\title{
Front Matter: Volume 10535
}

, "Front Matter: Volume 10535," Proc. SPIE 10535, Integrated Optics: Devices, Materials, and Technologies XXII, 1053501 (11 May 2018); doi: $10.1117 / 12.2323360$

SPIE. Event: SPIE OPTO, 2018, San Francisco, California, United States 


\title{
PROCEEDINGS OF SPIE
}

\section{Integrated Optics: Devices, Materials, and Technologies XXII}

\author{
Sonia M. García-Blanco \\ Pavel Cheben \\ Editors
}

29 January - 1 February 2018

San Francisco, California, United States

Sponsored and Published by

SPIE 
The papers in this volume were part of the technical conference cited on the cover and title page. Papers were selected and subject to review by the editors and conference program committee. Some conference presentations may not be available for publication. Additional papers and presentation recordings may be available online in the SPIE Digital Library at SPIEDigitalLibrary.org.

The papers reflect the work and thoughts of the authors and are published herein as submitted. The publisher is not responsible for the validity of the information or for any outcomes resulting from reliance thereon.

Please use the following format to cite material from these proceedings:

Author(s), "Title of Paper," in Integrated Optics: Devices, Materials, and Technologies XXII, edited by Sonia M. García-Blanco, Pavel Cheben, Proceedings of SPIE Vol. 10535 (SPIE, Bellingham, WA, 2018) Seven-digit Article CID Number.

ISSN: 0277-786X

ISSN: 1996-756X (electronic)

ISBN: 9781510615557

ISBN: 9781510615564 (electronic)

Published by

SPIE

P.O. Box 10, Bellingham, Washington 98227-0010 USA

Telephone +1 3606763290 (Pacific Time) · Fax +1 3606471445

SPIE.org

Copyright (c) 2018, Society of Photo-Optical Instrumentation Engineers.

Copying of material in this book for internal or personal use, or for the internal or personal use of specific clients, beyond the fair use provisions granted by the U.S. Copyright Law is authorized by SPIE subject to payment of copying fees. The Transactional Reporting Service base fee for this volume is $\$ 18.00$ per article (or portion thereof), which should be paid directly to the Copyright Clearance Center (CCC), 222 Rosewood Drive, Danvers, MA 01923. Payment may also be made electronically through CCC Online at copyright.com. Other copying for republication, resale, advertising or promotion, or any form of systematic or multiple reproduction of any material in this book is prohibited except with permission in writing from the publisher. The CCC fee code is 0277 $786 \mathrm{X} / 18 / \$ 18.00$.

Printed in the United States of America.

Publication of record for individual papers is online in the SPIE Digital Library.

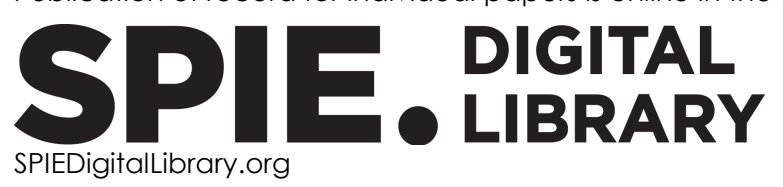

Paper Numbering: Proceedings of SPIE follow an e-First publication model. A unique citation identifier (CID) number is assigned to each article at the time of publication. Utilization of CIDs allows articles to be fully citable as soon as they are published online, and connects the same identifier to all online and print versions of the publication. SPIE uses a seven-digit CID article numbering system structured as follows:

- The first five digits correspond to the SPIE volume number.

- The last two digits indicate publication order within the volume using a Base 36 numbering system employing both numerals and letters. These two-number sets start with 00, 01, 02, 03, 04, 05, 06, 07, 08, 09, OA, OB ... OZ, followed by 10-1Z, 20-2Z, etc. The CID Number appears on each page of the manuscript. 


\title{
Contents
}

\author{
vii Authors \\ xi Conference Committee
}

\section{SESSION 1 INTEGRATED OPTICAL SENSORS}

1053504 High quality factor $\mathrm{Al}_{2} \mathrm{O}_{3}$ microring resonators for on-chip sensing applications [10535-3]

1053505 Design of ultra-compact composite plasmonic Mach-Zehnder interferometer for chemical vapor sensing [10535-4]

1053506 System for tracking femtometer resonance shifts of silicon photonics microring resonator by locking tunable laser [10535-5]

\section{SESSION 2 NONLINEAR PHOTONICS I}

1053508 SiNOI and AIGaAs-on-SOI nonlinear circuits for continuum generation in Si photonics [10535-7]

1053509 Hybrid ultra-high-Q silica microcavity Raman lasers (Invited Paper) [10535-8]

$10535 \mathrm{OB}$ Parallel periodically poled $\mathrm{LiNbO}_{3}$ waveguides module for polarization diversity wavelength conversion and amplification [10535-10]

\section{SESSION 3 NONLINEAR PHOTONICS II}

$10535 \mathrm{OE} \quad \mathrm{Hybrid}$ integration of $300 \mathrm{~nm}$-thick $\mathrm{LiNbO}_{3}$ films on ion-exchanged glass waveguides for efficient nonlinear integrated devices [10535-13]

10535 OF Optimal operation conditions for a push-pull dual-ring silicon modulator from a viewpoint of dispersion engineering and linearity (Invited Paper) [10535-55]

SESSION 4 SUBWAVELENGTH PHOTONICS AND METAMATERIALS

$10535 \mathrm{OH}$ Sub-wavelength multi-box waveguide-based label-free sensors (Invited Paper) [10535-15] SESSION 5 INTEGRATED ACOUSTO-OPTICS

10535 OK Brillouin lasers and amplifiers in silicon photonics (Invited Paper) [10535-18] 
10535 ON Composite material hollow core fibers: functionalisation with silicon and 2D materials [10535-21]

1053500 High-contrast slab waveguide fabrication in $\mathrm{KY}\left(\mathrm{WO}_{4}\right)_{2}$ by swift heavy ion irradiation [10535-22]

$105350 Q \quad$ Pedestal disk resonator in potassium yttrium double tungstate [10535-24]

\section{SESSION $7 \quad$ PHOTONIC INTEGRATION}

10535 OR Breaking the mold of photonic packaging (Invited Paper) [10535-25]

10535 OU A novel polishing stop for accurate integration of potassium yttrium double tungstate on a silicon dioxide [10535-27]

$10535 \mathrm{OV}$ Low temperature bonding of heterogeneous materials using $\mathrm{Al}_{2} \mathrm{O}_{3}$ as an intermediate layer [10535-28]

\section{SESSION $9 \quad$ MID IR}

1053512 Group IV mid-infrared devices and circuits (Invited Paper) [10535-35]

1053513 Development of integrated platform based on chalcogenides for sensing applications in the mid-infrared (Invited Paper) [10535-36]

$1053514 \quad$ Ultrafast laser-inscribed waveguides in IG2 chalcogenide glass for mid-infrared photonics applications [10535-37]

\section{SESSION 10 METROLOGY}

1053516 Comparative study of quantitative phase imaging techniques for refractometry of optical fibers [10535-39]

1053518 Multi-parameter estimation of high-Q silicon rich nitride resonators using optical frequency domain reflectometry [10535-41]

$105351 \mathrm{~A} \quad$ Uncertainty quantification and stochastic modelling of photonic device from experimental data through polynomial chaos expansion [10535-43]

\section{SESSION 11 INTEGRATED QUANTUM OPTICS}

10535 1B Quantum information processing with a travelling wave of light (Invited Paper) [10535-44] 
$105351 \mathrm{H} \quad$ Efficient coupling between $\mathrm{Si}_{3} \mathrm{~N}_{4}$ photonic and hybrid slot-based CMOS plasmonic waveguide [10535-50]

$105351 \mathrm{~J} \quad$ CMOS plasmonic waveguides co-integrated with LPCVD-based $\mathrm{Si}_{3} \mathrm{~N}_{4}$ via a butt-coupled interface [10535-52]

\section{SESSION $13 \quad$ PLANAR LIGHTWAVE CIRCUITS}

$105351 \mathrm{~K}$ Advanced modulation format using silicon modulators in the O-band (Invited Paper) [10535-53]

$10535 \mathrm{lL} \quad$ Reconfigurable integrated waveguide meshes for photonic signal processing and emerging applications (Invited Paper) [10535-54]

$105351 \mathrm{M} \quad$ O-band echelle grating demultiplexers on SiNOI featuring low-loss and reduced thermal sensitiveness [10535-56]

$105351 \mathrm{~N} \quad$ High-aggregate-capacity visible light communication links using stacked multimode polymer waveguides and micro-pixelated LED arrays [10535-57]

\section{SESSION 14 DIFFRACTIVES}

1053510 Thermally controlled Si photonic crystal waveguide slow light beam steering device [10535-58]

10535 IP Highly efficient grating couplers with mode conversion functions [10535-59]

10535 IR Temperature-dependence of polymer grating couplers for single-mode waveguides [10535-61]

\section{POSTER SESSION}

$105351 \mathrm{~T} \quad$ Hybrid plasmonic electro-optical absorption modulator based on epsilon-near-zero characteristics of ITO [10535-63]

$105351 \mathrm{U} \quad$ A waveguide loss measurement method based on the reflected interferometric pattern of a Fabry-Perot cavity [10535-64]

$105351 \mathrm{~W}$ Subwavelength focusing in the infrared range using a planar metallic lens of binary slits with refractive index modulation [10535-66]

$105351 Y \quad$ Precise core alignment in fabrication of polymer optical waveguides using the Mosquito method for three-dimensional optical circuits [10535-68]

1053520 All-silicon transparent conducting oxide-integrated electro-optical modulator [10535-70] 
1053521 Miniaturized semiconductor MOPA laser source at $772 \mathbf{n m}$ for the generation of UV laser light [10535-72]

1053522 Mid-infrared graphene-insulator-graphene plasmonic modulator [10535-73]

1053523 Compact broadband taper for low-loss coupling to a silicon nitride photonic wire [10535-75]

1053524 Mode converter using 2D MMI [10535-76]

1053525 True resolution enhancement for optical spectroscopy [10535-77]

1053527 Fabrication of high extinction ratio lithium niobate integrated optical modulators using photorefractive trimming [10535-79]

10535 2A Slow light modulator using semiconductor metamaterial [10535-82]

10535 2C Photocatalytic transformation in aerogel-based optofluidic microreactors [10535-84]

10535 2D Low chirp electroabsorption-modulated DFB laser fabricated by combining selective-area growth and double-stack active layer techniques [10535-85] 


\section{Authors}

Numbers in the index correspond to the last two digits of the seven-digit citation identifier (CID) article numbering system used in Proceedings of SPIE. The first five digits reflect the volume number. Base 36 numbering is employed for the last two digits and indicates the order of articles within the volume. Numbers start with 00, 01, 02, 03, 04, 05, 06, 07, 08, 09, 0A, 0B...0Z, followed by 10-1Z, 20-2Z, etc.

Abdelatty, Mohamed Y., 1T, 20 Abdel-Galil, Manar, IW Abe, Hiroshi, 10 Adelmini, L., 1M

Agruzov, P., 27

Armani, Andrea, 09

Baba, Toshihiko, 10

Badr, Mohamed M., 1T, 20

Baillieul, Marion, 13

Bamiedakis, N., $1 \mathrm{~N}$

Barbi, P., 06

Barwicz, Tymon, OR

Baudet, Emeline, 13

Baudot, C., 06, $1 \mathrm{~K}$

Baumgärtner, S., 21

Behunin, R. O., OK

Bélanger, Erik, 16

Belardi, W., ON

Bérubé, Jean-Philippe, 16

Bodiou, Loïc, 13

Bouchard, Aude, $\mathrm{OE}$

Boukerma, Kada, 13

Boyer, Nicolas, OR

Bramerie, L., IK

Brision, Stéphane, 08

Broquin, Jean-Emmanuel, $\mathrm{OE}$

Bru, Luis A., 18

Bruccoleri, M., 06

Bureau, Bruno, 13

Butcher, Helen L., 14

Caillaud, Celine, 13

Cao, W., 12

Capmany, Jose, $1 \mathrm{~L}$

Chaibi, M., $1 \mathrm{~K}$

Charrier, Joël, 13

Chatzianagnostou, E., 1H, 1J

Chen, Dongyu, 09

Cheung, Karen, $\mathrm{OH}$

Childers, Darrell, OR

Choi, Hyungwoo, 09

Chrostowski, Lukas, $\mathrm{OH}$

Colas, Florent, 13

Cooper, Justin T., 25

Cyr, Elaine, OR

Dabos, G., 1H, $1 \mathrm{~J}$

Dallery, J.-A., $1 M$

Date, Kumi, IY

Dattner, Yonathan, $\mathrm{OH}$

Dawson, M. D., IN

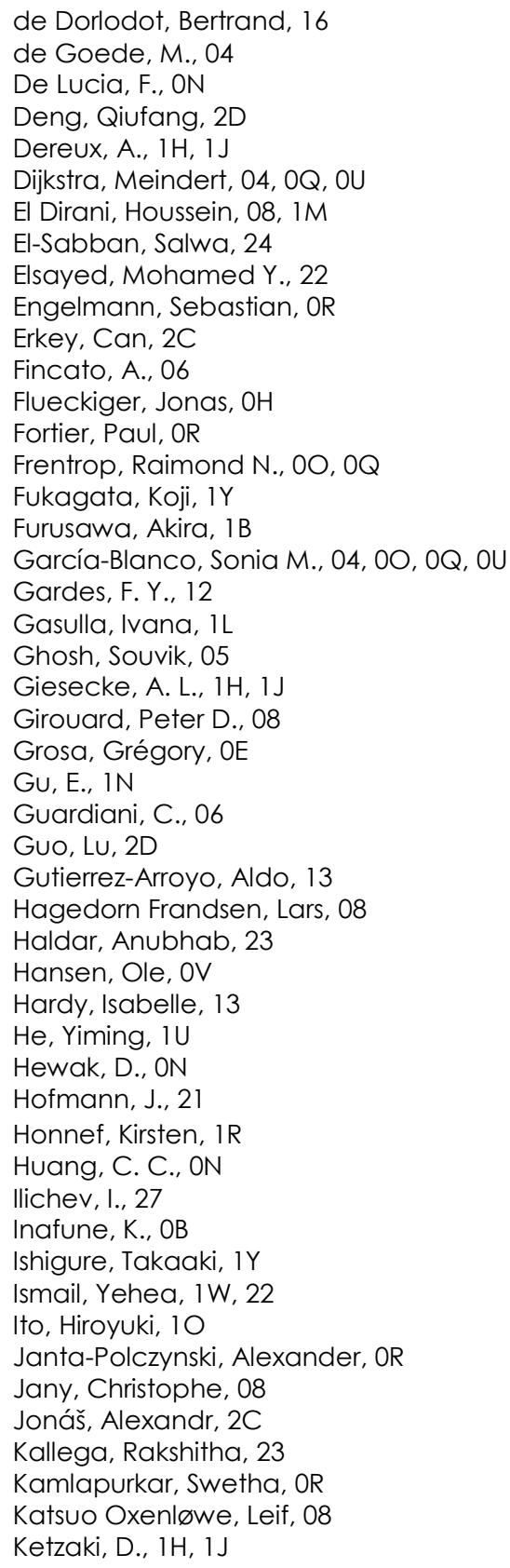


Khalil, Diaa, 24

Khater, Marwan, OR

Khokhar, A. Z., 12

Kimbrell, Eddie L., OR

Kiraz, Alper, 2C

Kishimoto, T., OB

Kittlaus, E., OK

Knights, Andrew P., OF

Kooijman, Roy, OU

Kovach, Andre, 09

Kumar, Shiva, OF

Laplatine, Loic, $\mathrm{OH}$

Lee, David, 14

Legrand, Louison, OE

Leidy, Robert, OR

Leisching, P., 21

Lemaitre, Jonathan, 13

Letartre, Xavier, 08

Lewis, A., ON

Li, Guifang, IP

Li, Pei, $1 R$

Li, Zhaosong, $1 \mathrm{U}$

Liang, Song, 2D

Lichoulas, Ted W., OR

Littlejohns, C. J., 12

Liu, Henan, $1 \mathrm{P}$

Louv, K., $1 \mathrm{M}$

LU, Dan, $1 \mathrm{U}$

Luan, Enxiao, $\mathrm{OH}$

MacLachlan, David G., 14

Manolis, A., 1H, $1 \mathrm{~J}$

Markey, L., 1H, $1 \mathrm{~J}$

Marquet, Pierre, 16

Marris-Morini, D., 1K

Martin, Yves, OR

Martínez, E., 04

Martinussen, Simen M., OQ, OU

Mashanovich, G. Z., 12

McKendry, J. J. D., 1N

Melati, Daniele, 1A

Melloni, Andrea, 06, 1A

Messaoudène, S., $1 \mathrm{~K}$

Michel, Karine, 13

Mittal, V., 12

Monat, Christelle, 08

$\mathrm{Mu}$, Jinfeng, OU

Muñoz, Pascual, 18

Murai, H., OB

Murugan, G. S., 12

Mushtaq, Zarlish, 1A

Nah, Jae-Woong, OR

Nazabal, Virginie, 13

Nedeljkovic, M., 12

Numata, Hidetoshi, OR

Obregón Núñez, R., 04

Ogawa, Hisashi, 1B

Ogawa, Y., OB

Oleske, Jeffrey B., 25

Olivares, José, 0O, 0Q

Olivier, Nicolas, 08
Olivier, S., 1M

Osman, A., 12

Ottaviano, Luisa, OV

Otterstrom, N., OK

Özbakır, Yaprak, 2C

Papaioannou, S., $1 \mathrm{~J}$

Parfenov, M., 27

Paschke, K., 21

Pastor, Daniel, 18

Peng, Bo, OR

Penty, R. V., IN

Pérez Galacho, D., IK

Perez, Daniel, $1 \mathrm{~L}$

Peserico, N., 06

Peucheret, C., $1 \mathrm{~K}$

Pleros, N., 1H, $1 \mathrm{~J}$

Poletti, F., ON

Porschatis, C., 1H, $1 \mathrm{~J}$

Pu, Minhao, 08

Qi, Y., 12

Qu, Z., 12

Rahman, B. M. A., 05

Rakich, P. T., OK

Ratner, Daniel, $\mathrm{OH}$

Reynolds, S., 12

Rezem, Maher, IR

Rinnert, Emmanuel, 13

Sahm, A., 21

Sahoo, Hitesh Kumar, OV

Sasaki, H., OB

Sazio, P. J. A., ON

Sciancalepore, Corrado, 08, 1M

Segerink, Frans, $0 Q$

Sekine, N., OB

Selvaraja, Shankar Kumar, 23

Semenova, Elizaveta, 08

Serikawa, Takahiro, 1B

Sethi, Purnima, 23

Shamray, A., 27

Shamray, L., 27

Shen, Xiaogin, 09

Sherman, Stanislav, IR

Shiozawa, Yu, 1B

Socquet-Clerc, C., $1 \mathrm{M}$

Soler Penades, J., 12

Stankovic, S., 12

Sun, Siwei, 2D

Swillam, Mohamed A., 1T, 1W, 20, 22

Taira, Yoichi, OR

Takanashi, Naoto, 1B

Takeda, Shuntaro, 1B

Takenobu, Shotaro, OR

Takeuchi, Goro, 10

Takeuchi, Moe, 10

Terada, Yosuke, 10

Thomson, D. J., 12

Thomson, Robert R., 14

Tormo-Márquez, Victoria, $00,0 Q$

Tronev, A., 27

Tsiokos, D., 1H, $1 \mathrm{~J}$ 
Vafapour, Zohreh, 2A

Vallée, Réal, 16

van Emmerik, Carlijn I., OU

Vivien, L., IK

Vulliet, N., 1K

Wageeh, Amr, 24

Wang, Wei, 2D

Wang, Zhao, OF

Waqas, Abi, 1 A

Weeber, J.-C., 1H, $1 \mathrm{~J}$

Weidmann, Damien, 14

White, I. H., 1N

Wilkinson, J. S., 12

Wilmart, Q., 1M

WU, Y., 12

Xie, E., $1 \mathrm{~N}$

Xie, Xiao, 2D

Ye, Zhichao, 18

Yoshikawa, Jun-ichi, 1B

Yun, Han, $\mathrm{OH}$

Yvind, Kresten, 08, 0V

Zappe, Hans, IR

Zhang, Lin, $1 \mathrm{P}$

Zhang, Meng, IP

Zheng, Yi, OV

Zhu, Hongliang, 2D

Zuffada, M., 06

Proc. of SPIE Vol. $105351053501-9$

Downloaded From: https://www.spiedigitallibrary.org/conference-proceedings-of-spie on 26 Apr 2023 Terms of Use: https://www.spiedigitallibrary.org/terms-of-use 
Proc. of SPIE Vol. 10535 1053501-10 Downloaded From: https://www.spiedigitallibrary.org/conference-proceedings-of-spie on 26 Apr 2023
Terms of Use: https://www.spiedigitallibrary.org/terms-of-use 


\title{
Conference Committee
}

\author{
Symposium Chairs
}

Connie J. Chang-Hasnain, University of California, Berkeley (United States)

Graham T. Reed, Optoelectronics Research Centre, University of Southampton (United Kingdom)

Symposium Co-Chairs

Jean-Emmanuel Broquin, IMEP-LAHC (France)

Shibin Jiang, AdValue Photonics, Inc. (United States)

Program Track Chair

Yakov Sidorin, Quarles \& Brady LLP (United States)

Conference Chairs

Sonia M. García-Blanco, University Twente (Netherlands)

Pavel Cheben, National Research Council Canada (Canada)

Conference Co-chairs

Jean-Emmanuel Broquin, IMEP-LAHC (France)

Gualtiero Nunzi Conti, Istituto di Fisica Applicata Nello Carrara (Italy)

Conference Program Committee

Pierre Berini, University of Ottawa (Canada)

Romeo Bernini, Istituto per il Rilevamento Elettromagnetico dell'Ambiente (Italy)

Alexandra Boltasseva, Purdue University (United States)

Xudong Fan, University of Michigan (United States)

Christoph M. Greiner, LightSmyth Technologies, Inc. (United States)

Robert Halir, Universidad de Málaga (Spain)

Robert A. Norwood, College of Optical Sciences, The University of Arizona (United States)

Alessia Pasquazi, University of Sussex (United Kingdom)

François Royer, Université Jean Monnet Saint-Etienne (France)

Jens H. Schmid, National Research Council Canada (Canada) 
Yakov Sidorin, Quarles \& Brady LLP (United States)

Avinoam Zadok, Bar-Ilan University (Israel)

Wei Zhou, Virginia Polytechnic Institute and State University (United States)

\section{Session Chairs}

1 Integrated Optical Sensors

Goran Z. Mashanovich, University of Southampton (United Kingdom)

2 Nonlinear Photonics I

Sonia M. García-Blanco, Universiteit Twente (Netherlands)

3 Nonlinear Photonics II

Sonia M. García-Blanco, Universiteit Twente (Netherlands)

4 Subwavelength Photonics and Metamaterials

Pavel Cheben, National Research Council Canada (Canada)

5 Integrated Acousto-Optics

Avinoam Zadok, Bar-Ilan University (Israel)

6 Novel Waveguide Technologies

Robert A. Norwood, College of Optical Sciences, The University of Arizona (United States)

7 Photonic Integration

Laurent Vivien, Centre de Nanosciences et de Nanotechnologies (France)

8 Novel Materials

Jean-Emmanuel Broquin, IMEP-LAHC (France)

9 Mid IR

Laurent Vivien, Centre de Nanosciences et de Nanotechnologies (France)

10 Metrology

Pavel Cheben, National Research Council Canada (Canada)

11 Integrated Quantum Optics

Jens H. Schmid, National Research Council Canada (Canada)

12 Plasmonics

Pierre Berini, University of Ottawa (Canada) 
13 Planar Lightwave Circuits

Avinoam Zadok, Bar-Ilan University (Israel)

14 Diffractives

David E. Zelmon, Air Force Research Laboratory (United States)

Proc. of SPIE Vol. $105351053501-13$

Downloaded From: https://www.spiedigitallibrary.org/conference-proceedings-of-spie on 26 Apr 2023 Terms of Use: https://www.spiedigitallibrary.org/terms-of-use 
Proc. of SPIE Vol. 10535 1053501-14 Downloaded From: https://www.spiedigitallibrary.org/conference-proceedings-of-spie on 26 Apr 2023
Terms of Use: https://www.spiedigitallibrary.org/terms-of-use 